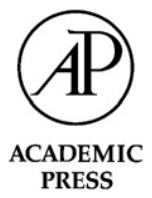

Available online at www.sciencedirect.com

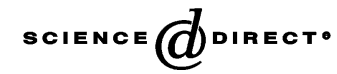

Journal of Economic Theory 109 (2003) 1-23

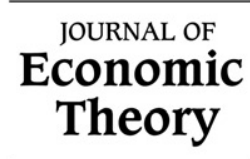

http://www.elsevier.com/locate/jet

\title{
Implementing efficient allocations in a model of financial intermediation
}

\author{
Edward J. Green ${ }^{\mathrm{a}}$ and Ping Lin ${ }^{\mathrm{b}, *}$ \\ ${ }^{a}$ Research Department, Federal Reserve Bank of Chicago, 230 South Lasalle St., \\ Chicago, IL 60604-0834, USA \\ ${ }^{\mathrm{b}}$ Department of Economics, Lingnan University, Tuen Mun, Hong Kong \\ Received 19 September 2000; final version received 15 January 2002
}

\begin{abstract}
In a finite-trader version of the Diamond and Dybvig (J. Polit. Econ. 91 (1983) 401) model, the ex ante efficient allocation is implementable by a direct mechanism (i.e., each trader announces the type of his own ex post preference) in which truthful revelation is the strictly dominant strategy for each trader. When the model is modified by formalizing the sequentialservice constraint (cf. Wallace (Fed. Reserve Bank Minneapolis Quart. Rev. 12 (1988) 3)), the truth-telling equilibrium implements the symmetric, ex ante efficient allocation with respect to iterated elimination of strictly dominated strategies.
\end{abstract}

(C) 2002 Elsevier Science (USA). All rights reserved.

JEL classification: D82; G21

Keywords: Financial intermediation; Bank run; Implementation

\section{Introduction}

This paper concerns the implementation of efficient allocations in a model of maturity transformation in financial structure. Maturity transformation is the financing of an intermediary's assets by liabilities (demand deposits at a bank, in particular) that are callable before the assets themselves mature. Bryant [1] shows that such a portfolio structure is a means of insuring the depositors against unobservable risks. He implicitly represents a bank as a rule or "allocation

\footnotetext{
*Corresponding author.

E-mail addresses: edward.green@chi.frb.org (E.J. Green), plin@ln.edu.hk (P. Lin).
} 
mechanism" that specifies the outcome, in each state of nature, of each possible profile of traders' decisions regarding whether or not to exercise the call options on their deposits. Bryant observes that maturity transformation is necessary in order to implement the symmetric, ex ante efficient allocation as a Bayesian Nash equilibrium. He also shows that some mechanisms that do implement that efficient allocation - notably the mechanism that most faithfully reflects the features of a bank-deposit contract in the context of his model-also can possess bank-run equilibria.

Diamond and Dybvig [4] address a set of issues related to Bryant. They study a model that brings the role of aggregate risk into sharp focus. They prove the following main results:

1. In environments without aggregate risk, demand deposit contracts can provide efficient risk-sharing. However, they can also produce bank runs.

2. In environments without aggregate risk, there is an allocation mechanism, suggested by historical banking regimes that have permitted suspension of convertibility of deposits when a "run" occurs, that implements the symmetric, ex ante optimal allocation in strictly dominant strategies. This is intuitively a particularly compelling notion of implementation that implies, among other things, that the Bayesian Nash equilibrium is unique. Obviously, then, there cannot be multiple, Pareto-ranked equilibria.

3. In some environments with aggregate risk, a deposit scheme with suspension of payments cannot implement the ex ante efficient allocation. However, If a government can exercise particular capabilities that the banking sector does not possess, then it is possible to implement the efficient allocation in Bayesian Nash equilibrium by establishing a deposit insurance scheme.

Regarding the last result, Diamond and Dybvig's analysis does not establish whether or not there is any allocation mechanism that implements the efficient allocation as a unique Bayesian Nash equilibrium. Wallace [11] provides a formalization of the sequential-service constraint to which previous researchers had appealed informally. He proves the following result. If the provision of deposit insurance is genuinely regarded as a feature of the overall allocation mechanism, and if it is this overall mechanism to which the sequential-service constraint applies, then deposit insurance is not feasible to provide. Taken together, these results raise the possibility that existence of a "bank-run" equilibrium might be an unavoidable problem for any mechanism that implements the efficient allocation as a Bayesian Nash equilibrium in an environment with aggregate risk.

In this paper, we reconsider the implementation problem in the Diamond and Dybvig environment. We use a finite-trader version of their model both to introduce aggregate risk in a natural and explicit way, and also to provide a formulation of the sequential-service constraint. We show that a naturally defined mechanism makes it a strictly dominant strategy for each trader to communicate his type truthfully, and that this dominant strategy equilibrium implements the symmetric, ex ante efficient allocation as a unique equilibrium outcome. This is in sharp contrast to Diamond 
and Dybvig's deposit-with-suspension mechanism which works in the absence, but not the presence, of aggregate risk. For our mechanism, the distinction between environments with and without aggregate risk is immaterial. We also consider the analogous allocation mechanism in environments with aggregate risk as well as a sequential-service constraint. We show that under the assumption that traders' utility functions exhibit non-increasing absolute risk aversion, for traders to truthfully communicate their types remains the unique strategy profile that survives iterated elimination of strictly dominated strategies. Thus, again, the mechanism has a unique Bayesian Nash equilibrium that possesses an intuitively compelling stability property, and the outcome of that equilibrium is the symmetric, ex ante efficient, equilibrium. Therefore, no "bank-run" equilibrium exists under the mechanisms we suggest.

We obtain different results from those of Diamond and Dybvig [4] because we consider a broader range of banking arrangements than they did. Specifically, they assumed that the banking arrangement must give all depositors who demand early withdrawals the same amount, namely the socially efficient amount based on the true value of the fraction of impatient depositors, no matter how many depositors actually claim to be impatient. In their model, although the consumption given to a trader varies with his own claimed type, the amount for each given type does not depend on the full information communicated to the bank by all traders. Instead of restricting attention to this type of "simple contracting", we allow the bank to utilize more fully the information reported by all depositors regarding their preferences. The banking arrangement in our model specifies consumptions for each trader of a given type under all possible configurations of reported types. In fact, for each vector of messages sent by the traders, our mechanism assigns traders the efficient allocation computed for the "reported economy". Allocation rules constructed this way turn out to be capable of preventing traders from lying about their types when they decide whether or not to make early withdrawals. Also, the mechanism of our model contains elements of a demand deposit contract: traders have the freedom to choose to either consume early (by claiming to be impatient) or wait to consume when their assets mature (by claiming to be patient). In light of our findings, Diamond and Dybvig's bank-run equilibrium appears to be an artifact of the simple contract modelling approach rather than a genuine feature of the economic environment that they have modelled.

Our analysis does not diminish the fundamental importance of Diamond and Dybvig's insight regarding financial instability, but we think that it shows the need to synthesize that insight with further ideas in order to understand financial instability fully. In light of the strikingly opposite results obtained in our model and the occurrence of bank runs in the history of the United States, one wonders what might prevent the individuals in the Diamond and Dybvig environment from using efficient mechanisms such as the one in our model. What would lead them instead to adopt the potentially destabilizing demand deposit contract considered in Diamond and Dybvig? Our results imply that environmental features from which Diamond and Dybvig's model abstracts are crucial to a full understanding of banking instability. In the concluding section, we reflect on our analysis to identify some candidates for such further, complementary ideas. 
Wallace [11] shows that the institutional arrangement of deposit insurance as modelled by Diamond and Dybvig [4] in the presence of aggregate risk is in fact infeasible in the presence of sequential-service constraint. Our formulation of sequential service is largely inspired by that of Wallace. In a recent important paper, Peck and Shell [10] also consider the implementation problem of the efficient allocation in the presence of sequential-service constraint and aggregate uncertainty. In contrast to our paper, Peck and Shell show that bank runs can still exist in equilibrium under the "optimal contract". As the authors pointed out, there are two important distinctions between Peck and Shell [10] and our model. First, impatient traders in Peck and Shell [10] are allowed to have utility functions that have higher marginal utility than that for patient traders, whereas both the patient and the impatient traders have the same form of utility functions in our model (and in [4] as well). Second, each trader in our model knows his clock time at which he arrives at the bank, while he does not know that in Wallace's or Peck and Shell's model environments.

The aggregate uncertainty in our model stems from the fact that there is a finite population of traders, each with an idiosyncratic preference shock. While this specification is a natural way to introduce aggregate uncertainty in an environment with a finite number of traders, it is different from the formulations in the original Diamond and Dybvig model and in later works such as Wallace [11,12], where aggregate uncertainty results from a change in the distribution of the taste shocks. We do not model this type of uncertainty that changes every trader's likelihood of becoming impatient.

\section{Basic model}

The economy consists of two time periods, time 0 and time 1 , and $I$ ex ante identical traders, $I<\infty$. Let $\mathbf{I}=\{1,2, \ldots, I\}$ be the set of traders. Each of the traders is uncertain about his preferences over consumption at date 0 and date 1 . With probability $P$, a trader becomes a type 0 agent in which case he derives utility from consumption at date 0 only. With probability $1-P$, the trader is a type 1 agent in which case he cares about consumptions at both dates. The set of possible states of nature can thus be represented by $\Omega=\{0,1\}^{I}$. Each of the traders learns his preferences at the beginning of date 0 . Let $\omega_{i}$ denote the realized type of trader $i$ in state $\omega \in \Omega$. As in Diamond and Dybvig [4], we assume that a trader's utility, $u_{i}$, is given by a function $v: \mathfrak{R}_{+} \rightarrow \mathfrak{R}$ of a consumption aggregate which includes consumption at both dates $\left(a_{0}, a_{1}\right)$ if $i$ is of type 1 , but which consists of consumption at date 0 alone if $i$ is of type 0 . That is,

$$
\forall i \quad \forall \omega \quad u_{i}(a, \omega)=v\left(a_{0}(i)+\omega_{i} a_{1}(i)\right) .
$$

Function $v$ is assumed to have the following properties:

Assumption 1. (i) $v$ is strictly increasing, continuously twice differentiable and strictly concave; (ii) $v$ satisfies the Inada conditions $\lim _{c \rightarrow 0} v^{\prime}(c)=\infty$ and $\lim _{c \rightarrow \infty} v^{\prime}(c)=0$; 
and (iii)

$\forall c \quad c v^{\prime \prime}(c) / v^{\prime}(c) \leqslant-1$ (relative risk aversion $\geqslant 1$ everywhere).

Each trader $i$ observes his own type only. Thus his information set, denoted by $\mathscr{E}_{i}$, is given by

$$
\mathscr{E}_{i}=\left\{\emptyset, \Omega,\left\{\omega \mid \omega_{i}=0\right\},\left\{\omega \mid \omega_{i}=1\right\}\right\} .
$$

There is an aggregate endowment of one unit of a good per person, which can be transformed into a consumption good available at either date 0 or date 1 . Whatever is not consumed at date 0 is augmented by a gross factor of $R>1$ at date 1 .

An ex post allocation is a function that specifies date 0 and date 1 consumptions for each trader. Given the endowment and the transformation technology, the set of feasible ex post allocations is

$$
\mathbf{A}=\left\{a: \mathbf{I} \rightarrow \mathfrak{R}_{+}^{2} \mid \sum_{i \in \mathbf{I}}\left[a_{0}(i)+R^{-1} a_{1}(i)\right] \leqslant I\right\} .
$$

A (feasible) state-contingent allocation is a $\mathscr{B}$-measurable function from $\Omega$ to A. Denote the set of such $\mathscr{B}$-measurable functions by $\mathbf{F}$. If $\vec{a} \in \mathbf{F}$ and $\omega \in \Omega$, then $\vec{a}(\omega)$ is the ex post allocation that the state-contingent allocation $\vec{a}$ specifies for state $\omega$.

Given the state-dependent utility function $u_{i}: \mathbf{A} \times \Omega \rightarrow \mathfrak{R}$, each trader $i$ maximizes the expectation of this function conditional on his type. Denote this conditional expectation by the function $U_{i}: \mathbf{F} \times \Omega \rightarrow \mathfrak{R}$, which is defined by $U_{i}\left(\vec{a}, \omega^{*}\right)=$ $E\left[u_{i}(\vec{a}(\omega), \omega) \mid \mathscr{E}_{i}\left(\omega^{*}\right)\right]$.

At the planning stage, the traders form a "bank" by choosing a mechanism in order to maximize the sum of all traders' expected utility. Without loss of generality, we consider direct revelation mechanisms under which each trader reports to the bank a message $m_{i} \in M \equiv\{0,1\}$. As a function of the state of the environment, then, trader $i$ 's message is an $\mathscr{E}_{i}$-measurable function $\mu_{i}: \Omega \rightarrow M$. This function $\mu_{i}$ will be called $i$ 's communication strategy. When each trader follows his communication strategy in state $\omega$, a profile $\mu(\omega)=\left(\mu_{1}(\omega), \ldots, \mu_{I}(\omega)\right)$ is generated which can be used as an informational basis for allocation. An allocation rule of a mechanism is a measurable function

$$
\alpha: \Omega \times M^{I} \rightarrow \mathbf{A} .
$$

An allocation rule $\alpha$ and a communication-strategy profile $\mu$ together determine a state-contingent allocation, denoted as $\alpha \circ \mu$, that takes each state $\omega$ to the ex post allocation $\alpha(\omega, \mu(\omega))$.

An equilibrium (specifically a Bayesian Nash equilibrium) of the allocation mechanism $(M, \alpha)$ is a communication-strategy profile $\mu^{*}$ such that, for any trader $i$ and any profile $\mu$ that $i$ can obtain by unilaterally changing his communication strategy while others' strategies remain the same, $U_{i}(\alpha \circ \mu, \omega) \leqslant U_{i}\left(\alpha \circ \mu^{*}, \omega\right)$ almost surely. 
Consider the allocation, $\vec{a}$, that, in each state of nature, maximizes the sum of traders' utilities, i.e.,

$$
\forall \omega \in \Omega \quad \sum_{i \in \mathbf{I}} u_{i}(\vec{a}(\omega), \omega)=\max _{\vec{a} \in \mathbf{F}(\omega)} \sum_{i \in \mathbf{I}} u_{i}(\vec{a}, \omega),
$$

where $\mathbf{F}(\omega)=\{f(\omega) \mid f \in \mathbf{F}\}$. This ex post optimal allocation also maximizes the sum of traders' expected utilities ex ante, as there is no reallocation of resources among different states of nature in this model. In the rest of the paper, and as in Diamond and Dybvig [4], we will first focus on the efficient allocation by assuming that the state of nature were observable, and then we show that such an allocation can be implemented in an incentive compatible way by a "banking contract."

\section{The case without sequential service}

In this section, we examine the mechanism design problem in the basic model assuming that the bank's resource distribution decision is made after all traders report their types to the bank. We will show that there exists a mechanism that implements the efficient allocation in this environment as an unique equilibrium.

Consider the problem of choosing $\vec{a} \in \mathbf{F}$ to maximize the sum of traders' expected utilities. By strict concavity of $v$, Jensen's inequality, and the fact that $R>1$ while consumption goods at the two dates are perfect substitutes for type- 1 traders, the following conditions should hold. In each state $\omega$, all type- 0 traders should receive identical consumption bundles $\left(c_{0}(\omega), 0\right)$ and all type-1 traders should receive identical consumption bundles $\left(0, c_{1}(\omega)\right)$. Let $\theta(\omega)=\sum_{i \in \mathbf{I}} \omega_{i}$ denote the number of patient traders in state $\omega$. Each efficient allocation $a=\vec{a}(\omega)$ should satisfy the following two equations (a first-order condition and a feasibility condition, respectively):

$$
v^{\prime}\left(c_{0}(\omega)\right)=R v^{\prime}\left(c_{1}(\omega)\right)
$$

and

$$
[I-\theta(\omega)] c_{0}(\omega)+R^{-1} \theta(\omega) c_{1}(\omega)=I
$$

These two equations determine $\vec{a}(\omega)$ uniquely. It is evident that $c_{0}(\omega)$ and $c_{1}(\omega)$ depend on $\omega$ only through $\theta(\omega)$. The following lemma explains the significance of the assumption regarding relative risk aversion in Assumption 1.

Lemma 1. Suppose that $v$ satisfies Assumption 1. Then, consumption level $c_{1}(\omega)$ of type-1 traders defined by (7) and (8) is a non-decreasing function of $\theta(\omega)$ More generally, let $\eta$ be a real variable taking values in $(0, I)$ and consider the problem of maximizing with respect to $\gamma$

$$
(I-\eta) v\left(\frac{\gamma}{I-\eta}\right)+\eta v\left(\frac{R(I-\gamma)}{\eta}\right)
$$


The solution, parametrized by $\eta$, is a function $\Gamma(\eta)$ that satisfies

$$
\frac{d}{d \eta} \frac{R(I-\Gamma(\eta))}{\eta} \geqslant 0 \text {. }
$$

Proof. To see that the general monotonicity assertion (10) implies the more specific assertion regarding $c_{1}$, note that if $0<\theta(\omega)=\eta<I$, then $c_{0}(\omega)=\Gamma(\eta) /(I-\eta)$ and $c_{1}(\omega)=R(I-\Gamma(\eta)) / \eta$ by (7) and (8). This equivalence can be extended to $\theta(\omega) \in\{0, I\}$, in view of the Inada conditions on $v$. (That is, defining $\Gamma(0)=I$ and $\Gamma(I)=0$ extends the definition of $\Gamma$ on $(0, I)$ continuously.)

Corresponding to (7), the first-order condition for (9) is

$$
v^{\prime}\left(\frac{\Gamma(\eta)}{I-\eta}\right)-R v^{\prime}\left(\frac{R(I-\Gamma(\eta))}{\eta}\right)=0 .
$$

Taking the derivative of (11) with respect to $\eta$ yields

$$
\begin{aligned}
& {\left[\Gamma^{\prime}(\eta)+\frac{\Gamma(\eta)}{I-\eta}\right] \quad \frac{v^{\prime \prime}(\Gamma(\eta) /(I-\eta))}{I-\eta}} \\
& \quad+R^{2}\left[\Gamma^{\prime}(\eta)+\frac{I-\Gamma(\eta)}{\eta}\right] \frac{v^{\prime \prime}(R(I-\Gamma(\eta)) / \eta)}{\eta}=0 .
\end{aligned}
$$

Now consider the derivative in (10).

$$
\frac{d}{d \eta} \frac{R(I-\Gamma(\eta))}{\eta}=\frac{-R}{\eta}\left[\Gamma^{\prime}(\eta)+\frac{I-\Gamma(\eta)}{\eta}\right] .
$$

In order to prove the lemma by establishing (10), then, it must be shown that $\Gamma^{\prime}(\eta)+\frac{I-\Gamma(\eta)}{\eta} \leqslant 0$. Note from (12) that $\Gamma^{\prime}(\eta)+\frac{I-\Gamma(\eta)}{\eta}$ and $\Gamma^{\prime}(\eta)+\frac{\Gamma(\eta)}{I-\eta}$ are either both zero or else they have opposite signs. Therefore, $\Gamma^{\prime}(\eta)+\frac{I-\Gamma(\eta)}{\eta} \leqslant 0$ is equivalent to

$$
\frac{I-\Gamma(\eta)}{\eta} \leqslant \frac{\Gamma(\eta)}{I-\eta}
$$

Inequality (14) follows from the assumption that $\forall c c v^{\prime \prime}(c) / v^{\prime}(c) \leqslant-1$. To see this, note that the assumption implies that $\frac{\partial}{\partial r}\left[r v^{\prime}(r s)\right] \leqslant 0$. This inequality and Eq. (11) imply that

$$
v^{\prime}\left(\frac{I-\Gamma(\eta)}{\eta}\right) \geqslant v^{\prime}\left(\frac{\Gamma(\eta)}{I-\eta}\right),
$$

which implies (14) by the concavity of $v$.

\subsection{A mechanism with a unique, efficient equilibrium}

Next we show that the efficient allocation determined by conditions (7) and (8) can be implemented as a truth-telling equilibrium of an allocation mechanism. In fact, the mechanism (defined in Theorem 1) possesses a property that truth-telling is the strictly dominant strategy for each trader. That is, whether a trader is of type 0 or type 1 , he receives a higher utility level from revealing his type truthfully to the bank 
than from misrepresenting it-regardless of what reports other traders give. This implies that the truth-telling equilibrium is the unique Bayesian Nash equilibrium of the mechanism. Therefore, no alternative, inefficient, "run" equilibrium of this mechanism can exist.

Theorem 1. Let $M=\{0,1\}$ be the set of reports for each trader. Define $x: M\{0, \ldots, I\} \rightarrow \mathfrak{R}$ by the conditions (analogous to (7) and (8)) that

$$
v^{\prime}(x(0, \eta))=R v^{\prime}(x(1, \eta))
$$

and

$$
[I-\eta] x(0, \eta)+R^{-1} \eta x(1, \eta)=I
$$

Define $\alpha: \Omega \times M^{I} \rightarrow \mathbf{A}$ by

$$
[\alpha(\omega, m)]_{i}=\left(\left(1-m_{i}\right) x\left(m_{i}, \sum_{j \in \mathbf{I}} m_{j}\right), m_{i} x\left(m_{i}, \sum_{j \in \mathbf{I}} m_{j}\right)\right) .
$$

The truthful communication strategy $\hat{\mu}_{i}(\omega)=\omega_{i}$ is the strictly dominant strategy for each trader $i$. The mechanism thus implements the efficient symmetric allocation in strictly dominant strategies, and consequently the profile of truthful communication strategies is its unique Bayesian Nash equilibrium.

Proof. Consider separately each of the two possible values of $\omega_{i}$. If $\omega_{i}=0$, then by (16) and (18), $i$ will receive a positive amount of consumption at date 0 if he sends message 0 , but will receive 0 consumption at date 0 if he sends message 1 . Because he derives utility only for consumption at date 0 , he strictly prefers to send message 0 rather than message 1 in state $\omega$.

Now suppose $\omega_{i}=1$. The strict concavity of $v$, together with (16), implies that

$$
x\left(1,0+\sum_{j \neq i} \mu_{j}(\omega)\right)>x\left(0,0+\sum_{j \neq i} \mu_{j}(\omega)\right)
$$

regardless of which communication strategies $\mu_{j}$ the other traders use. By (10) of Lemma 1 and the fundamental theorem of calculus,

$$
x\left(1,1+\sum_{j \neq i} \mu_{j}(\omega)\right) \geqslant x\left(1,0+\sum_{j \neq i} \mu_{j}(\omega)\right) .
$$

Therefore, trader $i$ must strictly prefer to send message 1, in which case he receives $x\left(1,1+\sum_{j \neq i} \mu_{j}(\omega)\right)$, rather than message 0 , in which case he receives $x\left(0, \sum_{j \neq i} \mu_{j}(\omega)\right)$. Thus, truth-telling is the strictly dominant strategy for trader $i$. By Myerson [9], a profile of strictly dominant strategies for a mechanism is the unique Bayesian Nash equilibrium of the mechanism. 


\subsection{An example: $v(c)=c^{1-\zeta} /(1-\zeta)$}

To illustrate the dominant strategy result in Theorem 1, consider the case where the traders' utility function is given by $v(c)=c^{1-\zeta} /(1-\zeta)$ where $\zeta>1$ is the relative risk aversion parameter. In this case, it is easily derived that the solution to Eqs. (7) and $(8)$ is

$$
c_{0}(\theta)=\frac{I}{I-\left(1-R^{\frac{1}{\zeta}-1}\right) \theta(\omega)} \text { and } c_{1}(\theta)=R^{\frac{1}{\zeta}} c_{0}(\theta) \text { for all } \omega \in \Omega,
$$

where $\theta(\omega)=\sum_{i \in \mathbf{I}} \omega_{i}$ is the number of patient traders in state $\omega$.

Two observations immediately follow. First, since $R>1, c_{1}(\theta)$ is greater than $c_{0}(\theta)$. The patient traders can take the advantage of the transformation technology so that they each receive more consumption than do the impatient traders in every state of nature. Secondly, both $c_{0}(\theta)$ and $c_{1}(\theta)$ strictly increase with $\theta$, the number of patient traders. The intuition for this is that as the number of impatient traders decreases, more endowment gets transformed to date 1 consumption, enabling both types of traders to consume more. These two properties imply that $c_{1}(\theta)>$ $c_{1}(\theta-1)>c_{0}(\theta-1)$ for all $\omega$. This in turn implies that truth-telling is the strictly dominant strategy for patient traders.

\section{Banking in an environment with sequential service}

The model of banking studied above abstracts from an important feature of an actual bank: that traders do not all contact the bank at the same time, and that the bank must deal promptly with traders who contact it early. The bank therefore is constrained from making its treatment of those traders contingent on information yet to be provided by later traders, especially if the early traders wish to make withdrawals. This feature plays an important role in Diamond and Dybvig's [4] intuitive discussion of their model, and it is formalized by Wallace [11] who derives further consequences from it. In view of the striking discrepancy between Theorem 1 and Diamond and Dybvig's analysis, and of the closer analogy between the theorem and Jacklin's [6] analysis that also abstracts from the sequential-service constraint, it is a salient question whether or not Theorem 1 can be extended to an environment with sequential service. Now we investigate this question and find an answer that is more or less in the affirmative. Specifically, if $v$ satisfies non-increasing absolute risk aversion as well as the conditions specified in Assumption 1, then the profile of truthful communication strategies is the unique profile that survives iterated elimination of strictly dominated strategies. It follows that, as in Theorem 1 , it is the unique Bayesian Nash equilibrium of the natural mechanism that implements the efficient allocation.

In the present formalization of the sequential-service constraint, every trader contacts the bank at some time during date 0 , these "arrival times" for different 
traders are stochastic and independently distributed, and each trader's arrival time is in his own information set. This last detail is crucial, for it implies that a trader who arrives very late can be almost certain that he is the last trader to arrive. We will show that, conditional on being last, truthful communication is the trader's unique utility-maximizing action. That is, any strategy that involves some untruthful communication by a trader when he arrives very late can be eliminated as being dominated by the strategy that agrees with it except at very late times, but that specifies truthful communication at those times. This result can then be "bootstrapped" to apply to communication at earlier times as well. Through this process of iterated elimination of strictly dominated strategies, we can show that the efficient allocation in the presence of a sequential-service constraint can be implemented as a unique, truth-telling equilibrium.

\subsection{Formalization of sequential service}

Modelling sequential service requires that the model in the previous section must be modified by enlarging the state space $\Omega$ to represent information about arrival times, and by making corresponding changes in the definitions of traders' types and of feasible allocations.

To enlarge the state space, let $\Omega^{\prime}=\{0,1\}^{I} \times[0,1]^{I}$, and $P \in(0,1)$. Assume that for all $i \leqslant I, \operatorname{Pr}\left(\omega_{i}=1\right)=P$, trader $i$ 's arrival time, denoted as $\omega_{I+i}$ is uniformly distributed on $[0,1]$; and that the projections of $\omega$ on its coordinates are independent r.v.'s. Replace the definition in (3) of trader $i$ 's type by

$$
\begin{aligned}
\mathscr{E}_{i}= & \left\{\left\{\omega \mid \omega_{i}=0 \omega_{I+i} \in B_{1}\right\}\right. \\
& \left.\cup\left\{\omega \mid \omega_{i}=1 \omega_{I+i} \in B_{2}\right\} \mid B_{1} \in \mathscr{F}, B_{2} \in \mathscr{F}\right\},
\end{aligned}
$$

where $\mathscr{F}$ is the $\sigma$-algebra of Borel sets on $[0,1]$. That is, in each state a trader knows his own utility function and his own arrival time at the bank, but he knows nothing about the other traders.

In order to formulate the sequential-service constraint, define the arrival-order statistics by $\tau:\{1, \ldots, I\} \times \Omega^{\prime} \rightarrow \mathbf{I}$. That is, $\tau(1, \omega), \tau(2, \omega), \ldots, \tau(I, \omega)$ are the first, second, ..., Ith traders in order of arrival determined by the coordinates $\omega_{I+1}, \omega_{I+2}, \ldots, \omega_{2 I}$ of $\omega$. Ties can be assumed to be broken arbitrarily in the zeroprobability event that several traders arrive simultaneously at the bank. Define the rank statistics $\rho: \mathbf{I} \times\{1, \ldots, I\} \times \Omega^{\prime} \rightarrow\{0, \ldots, I\}$, which are inverse to the order statistics in each state of nature, by $\rho(\tau(i, \omega), \omega)=i$.

Suppose that $\vec{a}=\left(\left(X_{0}^{1}, X_{1}^{1}\right), \ldots,\left(X_{0}^{I}, X_{1}^{I}\right)\right) \in \mathbf{A}^{\Omega^{\prime}}$ is an allocation. ${ }^{1}$ The intuitive content of the sequential-service constraint is that the mechanism represents a bank operating at a specific location that the trader visits at some time during date 0 . When trader $i$ visits, he communicates a message in $M=\{0,1\}$ determined by a communication strategy $\mu_{i}$ that is measurable with respect to $\mathscr{E}_{i}$, and he then receives

\footnotetext{
${ }^{1}$ In this section, since $\Omega^{\prime}$ is a continuum, $\mathbf{A}^{\Omega^{\prime}}$ denotes the set of Borel-measurable functions from $\Omega^{\prime}$ to $\mathbf{A}$.
} 
$X_{0}^{i}(\omega)$ immediately. ${ }^{2}$ This quantity thus must not depend on information from traders who arrive later in state $\omega$ than $i$ does, since those traders have not yet communicated their information to the bank. Since all traders are envisioned to arrive at the bank at some time before date 1 , when the consumption amounts $X_{1}^{i}(\omega)$ are distributed, those date-1 quantities are not analogously constrained.

That is, the amount $X_{0}^{\tau(1, \omega)}(\omega)$ of consumption given to trader $\tau(1, \omega)$ at date 0 must depend only on the identity of $\tau(1, \omega)$ and the time $\omega_{I+\tau(1, \omega)}$, both of which the bank observes, and on that trader's utility parameter $\omega_{\tau(1, \omega)}$, which he has the opportunity to communicate to the bank. (Whether or not he actually does communicate his utility parameter in equilibrium is irrelevant to the formulation of this constraint, which expresses the limitation imposed by the exogenous sequential nature of the opportunities for the bank to acquire information.) Next, the information that the bank can use to determine the date 0 consumption of the second trader to arrive consists of both this information about the first trader, which the bank remembers, and also the corresponding information about the second trader himself. And so forth. Formally, $\left(\left(X_{0}^{1}, X_{1}^{1}\right), \ldots,\left(X_{0}^{I}, X_{1}^{I}\right)\right)$ satisfies the sequential-service constraint if $\forall i$

$$
\begin{aligned}
X_{0}^{\tau(i, \omega)}= & E\left[X_{0}^{\tau(i, \omega)} \mid \tau(1, \omega), \ldots, \tau(i, \omega), \omega_{\tau(1, \omega)}, \ldots,\right. \\
& \left.\omega_{\tau(i, \omega)}, \omega_{I+\tau(1, \omega)}, \ldots, \omega_{I+\tau(i, \omega)}\right] .
\end{aligned}
$$

That is, the early consumption of a trader equals its expected value conditional on the history prior to his arrival at the bank. Thus, this trader's date-0 consumption does not depend on the events that would occur after he arrives at the bank.

In view of this constraint, the set of feasible state-contingent allocations $\mathbf{F}^{\prime}$ should be defined by

$$
\mathbf{F}^{\prime}=\left\{\vec{a} \mid \vec{a} \in \mathbf{A}^{\Omega^{\prime}} \text { and } \vec{a} \text { satisfies }(22)\right\} .
$$

\subsection{The efficient, symmetric, state-contingent allocation}

The optimization problem the bank faces now is

$$
\text { Maximize } \sum_{i \in \mathbf{I}} E\left[u_{i}(\vec{c}(\omega), \omega)\right] \text { subject to } \vec{c} \in \mathbf{F}^{\prime} \text {. }
$$

The key to solving the above problem is the observation, formalized in Lemma 2, that the arrival-order statistics $\tau(i, \omega)$ provide all of the relevant information about traders' arrival times. More precise arrival-time information is relevant neither to traders' enjoyment of utility nor to the technical feasibility of allocations in the sequential-service environment. ${ }^{3}$ In view of this observation, define mappings

\footnotetext{
${ }^{2}$ Here and throughout the description of the efficient allocation below, we assume that traders will report truthfully. Then, in Section 4.5, we show that there exists a mechanism that uses the allocation rule based on reported types and implements the full-information efficient allocation by inducing truth-telling behavior.

${ }^{3}$ Each trader will use his information about his precise arrival time to make inference about his probable rank in the arrival queue (which he does not observe directly) though, so this information is relevant to implementation.
} 


$$
\begin{gathered}
\sigma^{i}: \Omega^{\prime} \rightarrow\{0,1\}^{i} \text { for } 1 \leqslant i \leqslant I \text { by } \\
\forall j \leqslant i \quad \sigma_{j}^{i}(\omega)=\omega_{\tau(j, \omega)} .
\end{gathered}
$$

That is, $\sigma^{i}$ is the sequence of preference shocks of the first $i$ traders that have approached the bank in state $\omega$. Define the set of $0-1$ sequences of length at most $I$, including the null sequence, as $\mathscr{S}$. For $s \in \mathscr{S}$, let $\ell(s)$ denote the length of $s$. Define $\langle 0\rangle$ to be the sequence consisting of $I$ consecutive zeros. Define the weak and strict extension-ordering relations on $\mathscr{S}$ by

$$
\begin{array}{lll}
r \leqslant s \Leftrightarrow l(r) \leqslant l(s) & \text { and } \quad \forall i \leqslant l(r)\left[r_{i}=s_{i}\right], \\
r<s \Leftrightarrow l(r)<l(s) & \text { and } \quad \forall i \leqslant l(r)\left[r_{i}=s_{i}\right] .
\end{array}
$$

For example, if the economy consists of three traders, then space $\mathscr{S}$ will contain 14 elements. Among these 14 sequences, (0) and (1) represent, respectively, the cases that the trader arriving at the bank first is type 0 and type 1 . The (strict) extensions of $(0)$, for example, include $(0,0),(0,1),(0,0,0),(0,0,1),(0,1,0)$, and $(0,1,1)$. In general, the last component of a sequence $s \in \mathscr{S}$ represents the type of the trader who arrives at the bank at the end of that sequence. Sequential service constraint requires that the bank assigns a real number to each of such sequences as the date 0 consumption for the corresponding trader at the end of $s$. Date 1 consumption of a patient trader along a given sequence simply equals the remaining endowment, after all traders have arrived at the bank, augmented by $R$ and then equally divided among all the patient traders.

Define $\theta^{*}(s)=\sum_{i \leqslant \ell(s)} s_{i}$ and $\pi(s)=P^{\theta^{*}(s)}(1-P)^{\ell(s)-\theta^{*}(s)}$. That is, $\theta^{*}(s)$ is the number of patient traders along $s$ and $\pi(s)$ is probability that the types of the traders in $s$ have a $\left(\theta^{*}(s), \ell(s)-\theta^{*}(s)\right)$ split.

Lemma 2. Suppose that $\vec{a}=\left(\left(X_{0}^{1}, X_{1}^{1}\right), \ldots,\left(X_{0}^{I}, X_{1}^{I}\right)\right)$ solves problem (24). Then there exists a vector $x \in \mathfrak{R}_{+}^{\mathscr{S}}$ such that

$$
\sum_{r \leqslant\langle 0\rangle} x_{r}=I \quad \text { and } \forall s \in \mathscr{S}\left[\left[s_{l(s)}=1 \Rightarrow x_{s}=0\right] \text { and } \sum_{r \leqslant s} x_{r} \leqslant I\right]
$$

and, almost surely for all $i$,

$$
X_{j}^{\tau(i, \omega)}(\omega)=\left\{\begin{array}{ll}
x_{\sigma^{i}(\omega)} & \text { if } j=0 \text { and } \\
& \sigma_{i}^{i}(\omega)=\omega_{\tau(i, \omega)}=0, \\
\frac{R}{\theta^{*}\left(\sigma^{I}(\omega)\right)}\left(I-\sum_{r \leqslant \sigma^{I}(\omega)} x_{r}\right) & \text { if } j=1 \text { and } \\
0 & \sigma_{i}^{i}(\omega)=\omega_{\tau(i, \omega)}=1, \\
\text { otherwise. }
\end{array}\right\}
$$


If $\vec{a}$ and $x$ are related according to (28), then $^{4}$

$$
\begin{array}{rl}
\sum_{i \in \mathbf{I}} & E\left[u_{i}(\vec{a}(\omega), \omega)\right] \\
= & \sum_{\substack{l(s)=I \\
\theta^{*}(s)>0}} \pi(s)\left[\sum_{r \in z(s)} v\left(x_{r}\right)+\theta^{*}(s) v\left(\frac{R}{\theta^{*}(s)}\left(I-\sum_{q \leqslant s} x_{q}\right)\right)\right] \\
& +\pi(\langle 0\rangle) \sum_{r \leqslant\langle 0\rangle} v\left(x_{r}\right),
\end{array}
$$

where $z(s)=\left\{r \mid r \leqslant s\right.$ and $\left.r_{l(r)}=0\right\}$.

The first part of (27) states that the aggregate consumptions of all traders in the state that they are all impatient is equal to the total endowment. The second part of (27) states that patient agents should not consume at date 0 and that a non-negative amount of endowment should be stored for them for date 1 consumption. Condition (28) states that the last trader along a given sequence $\sigma^{i}(\omega)$ receives $x_{\sigma^{i}(\omega)}$ if he is impatient; and that if he is patient, then he receives at date 1 an amount equal to the total remaining resources equally divided among all the patient traders along the sequence implied by $\omega$. Expression (29) describes explicitly the terms in the expectation of the sum of traders utilities.

Proof. One can alternatively characterize $\vec{a}$ in terms of a vector of random variables $\left(\left(Y_{0}^{1}, Y_{1}^{1}\right), \ldots,\left(Y_{0}^{I}, Y_{1}^{I}\right)\right)$, where $Y_{j}^{i}(\omega)=X_{j}^{\tau(i, \omega)}(\omega)$ a.s. for each $i$ and $j$. Consider the state-contingent allocation $\vec{c}=\left(\left(Z_{0}^{1}, Z_{1}^{1}\right), \ldots,\left(Z_{0}^{I}, Z_{1}^{I}\right)\right)$, defined by $Z_{j}^{\tau(i, \omega)}(\omega)=$ $E\left[Y_{j}^{i}(\omega) \mid \sigma^{i}\right]$ a.s. It is easily verified that $\vec{c} \in \mathbf{F}^{\prime}$, and for every $i, E\left[u_{i}(\vec{a}(\omega), \omega)\right] \leqslant$ $E\left[u_{i}(\vec{c}(\omega), \omega)\right]$, with strict inequality for at least one $i$ if $\vec{a} \neq \vec{c}$. (This inequality must hold because $v$ is strictly concave and $\vec{c}$ is obtained by taking conditional expectation with respect to $\vec{a}$.) That is, $\vec{a} \neq \vec{c}$ would contradict the hypothesis of the lemma. By construction, $\vec{c}$ - that is to say, $\vec{a}$-can be characterized in terms of a vector $x \in \mathfrak{R}_{+}^{\mathscr{S}}$. This vector must actually satisfy (28), by the same considerations that prove the efficiency assertion for the case without sequential service. (Note that, in the context of (28), condition (27) states that traders of type 1 consume exclusively at date 1.) Condition (29) is verified by straightforward computation.

By Lemma 2, the solution to optimization problem (24) can be found by optimizing over a set of vectors in $\mathfrak{R}_{+}^{\mathscr{S}}$. Specifically, given the strict concavity of the right-hand side of (29), a solution is characterized by a vector that satisfies the firstorder conditions for optimization of (29) subject to constraints (27) and (28). That is, the following lemma holds.

\footnotetext{
${ }^{4}$ At the optimum, the sum of each trader's date- 0 and date- 1 consumptions is always positive for every state of nature, so expression (29) does not involve terms, such as $v(0)$, that might be infinite for some utility functions satisfying the assumptions of this model.
} 
Lemma 3. A necessary and sufficient condition for a state-contingent allocation $\vec{a}=$ $\left(\left(X_{0}^{1}, X_{1}^{1}\right), \ldots,\left(X_{0}^{I}, X_{1}^{I}\right)\right)$ to solve problem $(24)$ is that there should exist a vector $x \in \mathfrak{R}_{+}^{\mathscr{S}}$ that satisfies (27) and (28), and for all $r \in \mathscr{S}$ such that $r_{\ell(r)}=0$,

$$
\begin{aligned}
\pi(r) v^{\prime}\left(x_{r}\right)= & R \sum_{\substack{l(s)=I \\
\theta^{*}(s)>0 \\
r \leqslant s}} \pi(s) v^{\prime}\left(\frac{R}{\theta^{*}(s)}\left(I-\sum_{q \leqslant s} x_{q}\right)\right) \\
& +0^{\theta^{*}(r)} \pi(\langle 0\rangle) v^{\prime}\left(x_{\langle 0\rangle}\right) .
\end{aligned}
$$

That is, for any given sequence $r \in \mathscr{S}$, the consumption for the last trader who is at the end of $r$ and who is impatient should be chosen so as to balance his marginal utility of consumption against the sum of the expected marginal utility of all patient traders who arrive along all possible sequences that are to follow $r$. The last term on the right-hand side of (30) corresponds to the event that all $I$ traders turn out to be impatient. In that case, the last trader to arrive at the bank will consume whatever is left after resources have been distributed to traders who have arrived earlier: $x_{\langle 0\rangle}=$ $I-\sum_{r<\langle 0\rangle} x_{r}$. Of course, if $\theta^{*}(r)$ is already positive that term does not appear in the first-order condition.

\subsection{Iterated elimination of strictly dominated strategies}

Like Theorem 1, the corresponding result for the sequential-service environment will guarantee that the mechanism implementing the efficient allocation has a unique Bayesian Nash equilibrium. However, due to the sequential-service constraint, uniqueness cannot be established by the dominant-strategy argument used to prove Theorem $1 .^{5}$ Rather a concept of iterated elimination of strictly dominated strategies must be used. To state this concept, generalize the concept of strict dominance for an allocation mechanism $(M, \alpha)$ in the following way. First, for functions $f: \Omega^{\prime} \rightarrow \mathfrak{R}$ and $g: \Omega^{\prime} \rightarrow \Re$, define $f \prec g$ if $\operatorname{Pr}(f(\omega) \leqslant g(\omega))=1$ and $\operatorname{Pr}(f(\omega)<g(\omega))>0$. Next, for an arbitrary subset $K \subseteq\left(M^{\Omega^{\prime}}\right)^{I}$, define the relation $\prec_{i}^{K}$ on the strategy set $S_{i}$ of trader $i$ (that is, the subspace of $M^{\Omega^{\prime}}$ that is measurable with respect to $i$ 's information, $\omega_{i}$ and $\left.\omega_{I+i}\right)$ by specifying that $\phi \prec_{i}^{K} \phi^{\prime}$ when

$$
\forall \mu \in K, \forall \mu^{\prime} \in K\left[\begin{array}{l}
{\left[\phi=\mu_{i} \phi^{\prime}=\mu_{i}^{\prime} \text { and } \forall j \neq i \mu_{j}=\mu_{j}^{\prime}\right]} \\
\Rightarrow U_{i}(\alpha \circ \mu(\omega), \omega) \prec U_{i}\left(\alpha \circ \mu^{\prime}(\omega), \omega\right)
\end{array}\right]
$$

(with $U_{i}(\alpha \circ \mu(\omega), \omega)$ and $U_{i}\left(\alpha \circ \mu^{\prime}(\omega), \omega\right)$ being treated as unary functions of $\omega$ ).

Finally, define $M_{i}^{0}=S_{i}$ for each $i \leqslant I$, and for each $n \in \mathbf{N} \equiv\{0,1,2, \ldots\}$, let $K^{n}=$ $\prod_{j \leqslant I} M_{j}^{n}$ and define

$$
M_{i}^{n+1}=\left\{\phi \in M_{i}^{n} \mid \operatorname{not} \exists \phi^{\prime} \in M_{i}^{n} \phi \prec_{i}^{K^{n}} \phi^{\prime}\right\} .
$$

\footnotetext{
${ }^{5}$ Lin [8] shows this.
} 
The following lemma can be proved by an argument analogous to that in Myerson [9] regarding iterated elimination of strictly dominated strategies.

Lemma 4. For each $i$, let $M_{i}^{*}=\bigcap_{n \in \mathbf{N}} M_{i}^{n}$. If each $M_{i}^{*}$ contains a single element, then the unique element of $\prod_{i \leqslant I} M_{i}^{*}$ is the unique Bayesian Nash equilibrium of allocation mechanism $(M, \alpha)$.

\subsection{A monotonicity lemma}

The first-order condition (30) just derived for the sequential-service environment has analogous structure to the first-order condition (7) in the simultaneous communication environment studied in Section 3. The monotonicity assertion of Lemma 1, which provides the key to establishing Theorem 1 regarding the dominant strategy implementability of the efficient allocation in that environment, is proved by examining (7). A monotonicity result for a sequential-service environment is provable on the basis of Assumption 1, and it plays an analogous role to Lemma 1 in establishing implementability.

To understand intuitively the way that this monotonicity lemma will be formulated, it helps to know the order in which strictly dominated strategies will be eliminated. Essentially, that order is according to backward induction on the arrival time of a trader at the bank. We will establish that, if a trader arrives at the bank sufficiently late at date 0 , then he can be sure that everyone who arrives subsequently will give a truthful report, and that therefore the optimal report for the trader in question is also truthful. Here "sufficiently late" means "not before some time $t$," and we will show by working backward in time that $t$ can actually be taken to be zero. That is, truthful reporting is optimal for a trader regardless of what time he arrives at the bank.

Consider the case the last trader arrives at the bank. Prior to his arrival, $I-1$ traders had already visited the bank and a certain amount resource had been given to them. Let $y$ denote the amount of endowment remaining. The bank's decision problem in this case is simply as follows. If the last trader is patient, then he at date 1 will receive $R y /(\theta+1)$, his share of the remaining endowment transformed by the $R$-technology, where $\theta$ is the number of patient traders among the previous $I-1$ traders. If instead the last trader is impatient, then the bank needs to assign him positive consumption at date 0 , denoted by $\gamma_{I}$, by balancing his marginal utility against that of the $\theta$ patient traders. Thus, $\gamma_{I}$ satisfies the following: 6

$$
\gamma_{I}=\operatorname{Argmax}_{\gamma} v(\gamma)+\theta v\left(\frac{R(y-\gamma)}{\theta}\right) .
$$

The first-order condition is $v^{\prime}\left(\gamma_{I}\right)=R v^{\prime}\left[R\left(y-\gamma_{I}\right) / \theta\right]$ which implies $v^{\prime}\left(\gamma_{I}\right)>$ $\left.v^{\prime}\left[R\left(y-\gamma_{I}\right) / \theta\right)\right]$. Since $v^{\prime \prime}<0$, we have $\gamma_{I}<R y /(\theta+1)$. This simple observation has

\footnotetext{
${ }^{6}$ In the event that $\theta=0$, the last trader just consumes the undistributed endowment so $\gamma_{I}=y$.
} 
an important implication regarding the communication strategy for the last trader. If he is impatient, then the last trader surely does not want to claim to be patient because he does not value date 1 consumption. If he is patient, then he receives $R y /(\theta+1)$ if he tells the truth which is greater than $\gamma_{I}$, the amount he will receive if he lies. Thus, the trader who arrives the last never lies about his type.

Now consider the situation of a trader $i$ who arrives at the bank at some time during date 0 after $I-J-1$ traders have already arrived, and before the last $J$ traders will arrive, and who knows that those $J$ traders who follow him will give truthful reports. Suppose that the $I-J-1$ traders who arrive prior to trader $i$ have given a vector of reports $p \in \mathscr{S}$, with $\ell(p)=I-J-1$. Some non-negative amount of the endowment good will have already been given to the earlier traders who have reported themselves to be of type 0 , and an amount $y=y(p)$ remains to be allocated.

In terms of a representation like the one developed in Lemma 2, the bank must allocate consumption in a way specified by a vector $\gamma \in \mathfrak{R}_{+}^{\mathscr{S}^{\prime}}$, where $\mathscr{S}^{\prime}=$ $\{s \in \mathscr{S} \mid \ell(s) \leqslant J\}$. If $i$ reports being of type 0 , then the optimization problem of the bank is to maximize ${ }^{7}$

$$
\begin{aligned}
& v\left(\gamma_{\varnothing}\right)+\sum_{\ell(s)=J} \pi(s)\left[\left(\sum_{r \in z(s)} v\left(\gamma_{r}\right)\right)+\left(\theta^{*}(p)\right.\right. \\
& \left.\left.\quad+\theta^{*}(s)\right) v\left(\frac{R}{\theta^{*}(p)+\theta^{*}(s)}\left(y(p)-\sum_{q \leqslant s} \gamma_{q}\right)\right)\right] .
\end{aligned}
$$

(The second term in the bracket is taken to be zero if $\theta^{*}(p)=\theta^{*}(s)=0$, since in that case there is no trader who wishes to consume at date 1.)

If $i$ reports being of type 1 , then the maximization problem is to optimize

$$
\begin{aligned}
& \sum_{\ell(s)=J} \pi(s)\left[\left(\sum_{r \in z(s)} v\left(\gamma_{r}\right)\right)\right. \\
& \left.+\left(\theta^{*}(p)+\theta^{*}(s)+1\right) v\left(\frac{R}{\theta^{*}(p)+\theta^{*}(s)+1}\left(y(p)-\sum_{q \leqslant s} \gamma_{q}\right)\right)\right] .
\end{aligned}
$$

The solutions to the above two optimization problems depend on $p$ (and $y(p)$ ), and are denoted as $\left\{\Gamma_{s}(0, p)\right\}_{s \in \mathscr{S}^{\prime}}$ and $\left\{\Gamma_{s}(1, p)\right\}_{s \in \mathscr{C}^{\prime}}$, respectively. ${ }^{8}$

\footnotetext{
${ }^{7}$ The optimization problem yields consumption for each sequence $q$ that follows trader $i . \gamma_{\varnothing}$ represents the date 0 consumption of the trader $i$ in question if he claims to be impatient and $\gamma_{q}$ represents the date 0 consumption assigned to the impatient trader who arrives at the end of sequence $q$.

${ }^{8}$ For the special case that there are three traders each having utility function $v(c)=c^{1-\varsigma} /(1-\varsigma)$, Green and Lin [5] explicitly solved for the efficient allocation. The purpose of that paper is to illustrate concretely the treatment of the sequential-service constraint analyzed in the present general model.
} 
Analogously to what we have done in Lemma 1, these formulae can be subsumed in a general formula. Specifically, they correspond to $\eta=0$ and $1 \mathrm{in}^{9}$

$$
\begin{aligned}
& (1-\eta) v\left(\frac{\Gamma_{\varnothing}(\eta, p)}{1-\eta}\right)+\sum_{\ell(s)=J} \pi(s)\left[\left(\sum_{r \in z(s)} v\left(\Gamma_{r}(\eta, p)\right)\right)\right. \\
& \left.+\Phi \cdot v\left(\frac{R}{\Phi}\left(y(p)-\sum_{q \leqslant s} \Gamma_{q}(\eta, p)\right)\right)\right],
\end{aligned}
$$

where $\Phi \equiv \theta^{*}(p)+\theta^{*}(s)+\eta$. Denote the solution as $\Gamma_{r}(\eta, p)$.

Our goal is to prove the following statement, which formalizes the idea that a trader of type 1 should reveal his type truthfully if all traders who will arrive at the bank after him are going to reveal their types truthfully.

Lemma 5. For each $\eta \in[0,1]$, let $\Gamma(\eta, p) \in \mathfrak{R}_{+}^{\mathscr{S}^{\prime}}$ maximize (34) subject to the constraint (27) (with $\mathscr{S}^{\prime}$ and $y(p)$ replacing $\mathscr{S}$ and $I$, respectively, in the statement of the constraint). Suppose that $v$ satisfies Assumption 1 and also the condition that

$$
\begin{aligned}
& \forall c \quad \frac{d}{d c} \frac{v^{\prime \prime}(c)}{v^{\prime}(c)} \geqslant 0 \text { (absolute risk aversion is } \\
& \text { non-increasing everywhere). }
\end{aligned}
$$

Then, for any $p$,

$$
v\left(\Gamma_{\varnothing}(0, p)\right)<\sum_{\ell(s)=J} \pi(s) v\left(\frac{R}{\theta^{*}(p)+\theta^{*}(s)+1}\left(y-\sum_{q \leqslant s} \Gamma_{q}(1, p)\right)\right) .
$$

Proof. See the appendix.

If trader $i$ who arrives following a given sequence $p$ of length of $I-J-1$ is of type 0 , he receives $\Gamma_{\varnothing}(0, p)$ at date 0 . If he is type 1 , then he receives zero date 0 consumption but his date 1 consumption depends the types of traders who arrive after him. Along a sequence $(p, s)$ that follows $p, \theta^{*}(s)$ of such $J$ remaining traders will be impatient, in which case $\sum_{q \leqslant s} \Gamma_{q}(1, p)$ will be given to them at date 0 . At date 1 , then, the remaining resource will be divided equally among trader $i$ and all other patient traders. Summing up over all such possible sequences $(p, s)$, the above lemma says the efficient allocation has the property that the expected utility of trader $i$ is higher if he reports to be patient. Therefore, trader $i$ never wants to lie about his type.

\footnotetext{
${ }^{9}$ By the Inada conditions, $\lim _{x \rightarrow \infty} v(x) / x=0$. So the first term in (34) is zero when $\eta=1$.
} 


\subsection{A mechanism with a unique, efficient equilibrium}

In light of Lemma 5, we define a mechanism as follows. Let $x: \mathscr{S} \rightarrow \mathfrak{R}_{+}$be the vector satisfying the optimality conditions, (27), (28), and (30). Let $M=\{0,1\}$ be the set of reports for each trader. Define $\alpha: \Omega^{\prime} \times M^{I} \rightarrow \mathbf{A}$ by

$$
\begin{aligned}
{[\alpha(\omega, m)]_{i}=} & \left(\left(1-m_{i}\right) x\left(m_{\tau(1, \omega)}, \ldots, m_{\tau(\rho(i, \omega), \omega)}\right),\right. \\
& \left.m_{i} \frac{R}{\sum_{j \leqslant I} m_{j}}\left(I-\sum_{j \leqslant I} x\left(m_{\tau(1, \omega)}, \ldots, m_{\tau(j, \omega)}\right)\right)\right) .
\end{aligned}
$$

Theorem 2. Suppose that $v$ satisfies Assumption 1 and (35). Then the profile of truthfulcommunication strategies $\hat{\mu}_{i}(\omega)=\omega_{i}$ is the unique profile that survives iterated elimination of strictly dominated strategies under the above mechanism. The mechanism thus implements the symmetric, ex ante efficient allocation by a unique Bayesian Nash equilibrium.

The proof of the theorem follows almost immediately from the "backward induction" reasoning made in the previous subsection. However, since traders observe their own respective arrival times only, they do not know their exact positions in a given sequence of arrivals. So the backward induction reasoning does not guarantee automatically the result in Theorem 2. But as argued earlier, a trader who arrives the bank sufficiently late can be practically sure that he is the last trader to arrive. So he will tell the truth in communicating with the bank. Working backward from the end of date 0 to the beginning, the proof below establishes the result in Theorem 2.

Proof. For all $i \leqslant I$ and all $t \leqslant 1$, define $S_{i}^{t}=\left\{\mu \in S_{i} \mid \forall \omega \omega_{I+i} \geqslant t \Rightarrow \mu(\omega)=\omega_{I+i}\right\}$, where $S_{i}$ is the set of $\mathscr{E}_{i}$-measurable functions $\mu_{i}: \Omega^{\prime} \rightarrow M$. That is, $S_{i}$ is the space of trader $i$ 's reporting strategies as functions of $\omega$, and $S_{i}^{t}$ is the collection of such strategy functions that involve truthful reporting if trader $i$ is to arrive at the bank at $t$ or later.

Define

$$
\begin{aligned}
V(p)= & \sum_{\ell(s)=I-\ell(p)-1} \pi(s) v\left(\frac{R}{\theta^{*}(p)+\theta^{*}(s)+1}\left(y(p)-\sum_{q \leqslant s} \Gamma_{q}(1, p)\right)\right) \\
& -v\left(\Gamma_{\varnothing}(0, p)\right) .
\end{aligned}
$$

By Lemma $5, V(p)>0$ for all $p \in \mathscr{S}$. Let $V^{*}=\min _{p \in \mathscr{S}} V(p)$. Since $\mathscr{S}$ is finite, $V^{*}>0 . V^{*}$ represents the minimum gain for a patient trader from reporting being patient rather than impatient, among all possible sequences of preceding reports by other traders, assuming all subsequent reports are truthful. 
Also define

$$
\begin{aligned}
W^{*}= & \min _{p \in \mathscr{S}, \ell(s)=I-\ell(p)-1} v\left(\frac{R}{\theta^{*}(p)+\theta^{*}(s)+1}\left(y(p)-\sum_{q \leqslant s} \Gamma_{q}(1, p)\right)\right) \\
& -v\left(\Gamma_{\varnothing}(0, p)\right) .
\end{aligned}
$$

$W^{*}$ represents the worst change in utility, for a patient trader from reporting being patient rather than impatient, among all possible sequences of preceding and subsequent reports by other traders. Thus $W^{*}$ is a lower bound for the change in expected utility from truthfully reporting being patient rather than reporting being impatient, regardless of what reporting strategies the other traders adopt. Again because $\mathscr{S}$ is finite, $W^{*}>-\infty$.

Consider the case that trader $i$ arrives at time $t$ that is very close to 1 . The probability that trader $i$ is the last trader to arrive is $t^{I-1}$, which approaches 1 as $t \rightarrow 1$. Conditioning on being the last one to arrive, reporting truthfully yields higher utility for trader $i$, by Lemma 5 . Thus, since $W^{*}>-\infty$, the unconditional expected utility from reporting being patient is strictly greater than from reporting being impatient if $t$ is sufficiently close to 1 . Let $t_{0}<1$ be sufficiently close to 1 that it is strictly better for $i$ to report being patient if $\omega_{I+i} \geqslant t_{0}$. Thus, every trader $j \in \mathbf{I}$ will report truthfully if he arrives at the bank at time $t_{0}$ or later (i.e., that $\omega_{I+j} \geqslant t_{0}$ ).

Now consider $t \leqslant t_{0}$, and suppose all traders who arrive at $t$ or later will report truthfully. Let $\varepsilon>0$ and suppose that trader $i$ arrives at the bank at time $t-\varepsilon$. Then the probability that all subsequent traders will arrive at $t$ or later is at least $[(1-$ $t) /(1-(t-\varepsilon))]^{I-1}$. The gain in expected utility for $i$ from truthful reporting of being patient rather than untruthful reporting of being impatient is at least

$$
\begin{aligned}
g(t, \varepsilon) & \equiv[(1-t) /(1-(t-\varepsilon))]^{I-1} V^{*} \\
& +\left[1-[(1-t) /(1-(t-\varepsilon))]^{I-1}\right] W^{*}
\end{aligned}
$$

As $\varepsilon \rightarrow 0, g(t, \varepsilon) \rightarrow V^{*}>0$ for all $t \leqslant t_{0}$.

Now consider the set $K=\left\{(t, \varepsilon) \mid 0 \leqslant t-\varepsilon \leqslant t \leqslant t_{0}\right\}$. Since $g$ is continuous and $K$ is compact, $g$ is uniformly continuous on $K$. Thus, there exists $\varepsilon_{0}>0$ such that, for all $(t, \varepsilon) \in\left(\left[0, t_{0}\right] \times\left[0, \varepsilon_{0}\right]\right) \cap K, g(t, \varepsilon)>V^{*} / 2>0$. Thus, $i$ maximizes expected utility uniquely by reporting truthfully that he is patient if he arrives at $t-\varepsilon_{0}$ or later, if all other traders arriving at $t$ or later will report truthfully.

Let $t_{n}=t_{0}-(n-1) \varepsilon_{0}, n=1,2, \ldots$. By the above arguments, for each $i$ the set $S_{i}^{*}=\bigcap_{n \in \mathbf{N}} S_{i}^{t_{n}}$ contains a single element, namely, $\mu(\omega)=\omega_{i}$ for all $\omega \in \Omega^{\prime}$. By Lemma 4, the unique element in $\prod_{i \leqslant I} S_{i}^{*}$ is the unique Bayesian Nash equilibrium of allocation mechanism $(M, \alpha)$. 


\section{Conclusion}

In a finite-trader version of the Diamond-Dybvig [4] model with sequential-service constraint, we have analyzed the question of maturity transformation. Like Diamond and Dybvig, the (direct revelation) mechanism in our model implements the symmetric, ex ante efficient allocation as a truth-telling equilibrium. Unlike Diamond and Dybvig, however, our mechanism, which does not involve government intervention (e.g., deposit insurance) of any sort, has no "bank-run" equilibrium. There are two reasons that our mechanism does not posses a bank-run equilibrium. First, the banking contract in our model is flexible in that the consumption given to a particular trader depends on the number of traders who have claimed to be impatient. Because of this, the amount to be given to a patient trader is never zero in any state of nature. Thus, the flexible contract is designed in such a way that the bank never runs out of resource. The second reason for the absence of a bank run in our model is that traders can correctly forecast that traders arriving late will behave truthfully. This allows the traders to perform the backward induction reasoning and be able to eliminate the strictly dominated strategies. The ability of traders to observe the clock time of their arrivals is a crucial formal assumption for our implementation result.

Diamond and Dybvig's model has been interpreted as an explanation of the numerous bank runs observed in US history. The force of the result proved here is that traders in a similar model environment need not tolerate the possibility of runs in order to adopt a mechanism that guarantees ex ante, interim, and ex post Pareto efficiency. That is, Diamond and Dybvig's explanation needs to be completed by reference to some feature of the historical US banking environment that would make such a mechanism infeasible. Let us cite two examples of the numerous features that are candidates to play this role.

One obvious feature of an actual banking system that the Diamond and Dybvig model, as well as ours, fails to capture is its ongoing nature. If the population of the economy has an overlapping generation structure, for example, then no trader is the last one to arrive at the bank as the backward induction methodology in our analysis would require. The same problem would arise if the size of the population is not observable to individual traders so that no trader is certain whether or not he is almost the last one in line. ${ }^{10}$ When these features are present, the validity of our "norun" result needs to be reconsidered.

Another feature of a real banking industry which is absent in our model is the incentive problem of banking executives whose objectives may be different from that of a social planner. Suppose that the mechanism in our model is run by a banker, instead of the planner. The traders report their realized types to the banker who then distributes the resources to the traders, supposedly in the way specified by the mechanism. But, if the traders cannot observe each other's reports, then there is no guarantee that the banker allocates the resources based on the true reported state of nature. The banker might keep part of the endowment for his own consumption and

\footnotetext{
${ }^{10}$ Strictly speaking, the population-size distribution must have infinite support and a "fat tail."
} 
then claim that a great deal of resources has been withdrawn by a number of impatient traders that is larger than is actually the case. Anticipating this possibility and its consequence that less resources will be available at time 1, the patient traders may be tempted to withdraw early, increasing the likelihood of a bank run. Such an incentive problem might be another explanation of why the banking contract in our model is not observed, and why runs have historically occurred. ${ }^{11}$

Finally, we note another direction for future research. Under the treatment of the sequential-service constraint in our model, each trader's arrival time at the bank is governed by an exogenous stochastic process. This simple formulation helps clarify the structure of the optimization problem facing the bank. Alternatively, one can consider the case that traders' preferences are realized in a sequential order, rather than simultaneously as in the present model. After learning about their types, the traders then decide whether or not to make early withdrawals by approaching the bank. In such a case, a trader can contact the bank and demand early withdrawals even before he learns about his type. Also, after observing his type, a patient trader can either wait until date 1 to consume or go to the bank to demand early consumption anytime before date 1 , if he observes "too many" traders coming to the bank. Therefore, arrival times are also a part of a trader's strategy, along with reporting types to the bank. Extending the present model along this direction deserves further study.

\section{Appendix}

Proof of Lemma 5. To streamline notation, we drop the argument $p$ in $\Gamma(\eta, p)$ and simply write it as $\Gamma(\eta)$. The function $\Gamma$ satisfies the following first-order condition at each value of $\eta \in(0,1)$.

$$
0=\left\{\begin{array}{cc}
v^{\prime}\left(\frac{\Gamma_{\varnothing}(\eta)}{1-\eta}\right)-R \sum_{\ell(s)=J} \pi(s) & \\
v^{\prime}\left(\frac{R}{\theta^{*}(p)+\theta^{*}(s)+\eta}\left(y-\sum_{q<s} \Gamma_{q}(\eta)\right)\right) & \text { FOC for } \Gamma_{\varnothing} \\
\pi(r) v^{\prime}\left(\Gamma_{r}(\eta)\right) & \\
-R \sum_{\substack{\ell(s)=J \\
r \leqslant s}} \pi(s) v^{\prime}\left(\frac{R}{\theta^{*}(p)+\theta^{*}(s)+\eta}\right. & \\
\left.\times\left(y-\sum_{q \leqslant s} \Gamma_{q}(\eta)\right)\right) & \text { FOC for } \Gamma_{r} \gamma>\varnothing .
\end{array}\right\}
$$

This condition implies the following three martingale-marginal-utility equations:

$$
v^{\prime}\left(\frac{\Gamma_{\varnothing}(\eta)}{1-\eta}\right)=\sum_{\ell(s)=1} \pi(s) v^{\prime}\left(\Gamma_{s}(\eta)\right)
$$

\footnotetext{
${ }^{11}$ Diamond [3] and Krasa and Villamil [7] examined the issue of monitoring in the context of financial intermediation. Calomiris and Kahn [2] showed that demand deposit contract can serve as a device to monitor bank managers.
} 
for $0<\ell(r)<J$ and $r_{\ell(r)}=0$,

$$
\pi(r) v^{\prime}\left(\Gamma_{r}(\eta)\right)=\sum_{\ell(s)=\ell(r)+1} \pi(s) v^{\prime}\left(\Gamma_{s}(\eta)\right)
$$

and, for $\ell(r)=J$ and $r_{J}=0$,

$$
v^{\prime}\left(\Gamma_{r}(\eta)\right)=R v^{\prime}\left(\frac{R}{\theta^{*}(p)+\theta^{*}(s)+\eta}\left(y-\sum_{q \leqslant r} \Gamma_{q}(\eta)\right)\right) .
$$

Since $R>1$, Eq. (A.1) implies that

$$
v^{\prime}\left(\Gamma_{\varnothing}(0)\right)>\sum_{\ell(s)=J} \pi(s) v^{\prime}\left(\frac{R}{\theta^{*}(p)+\theta^{*}(s)}\left(y-\sum_{q<s} \Gamma_{q}(0)\right)\right) .
$$

By an argument analogous to Lemma 1, involving the martingale conditions (A.2)(A.4),

$$
\frac{d}{d \eta}\left[\sum_{\ell(s)=J} \pi(s) v^{\prime}\left(\frac{R}{\theta^{*}(p)+\theta^{*}(s)+\eta}\left(y-\sum_{q \leqslant s} \Gamma_{q}(\eta)\right)\right)\right] \leqslant 0 .
$$

By the fundamental theorem of calculus and continuity at the endpoints 0 and 1 , (A.6) implies that

$$
\begin{aligned}
& \sum_{\ell(s)=J} \pi(s) v^{\prime}\left(\frac{R}{\theta^{*}(p)+\theta^{*}(s)}\left(y-\sum_{q \leqslant s} \Gamma_{q}(0)\right)\right) \\
& \geqslant \sum_{\ell(s)=J} \pi(s) v^{\prime}\left(\frac{R}{\theta^{*}(p)+\theta^{*}(s)+1}\left(y-\sum_{q<s} \Gamma_{q}(1)\right)\right) .
\end{aligned}
$$

Eqs. (A.5) and (A.7) imply that

$$
v^{\prime}\left(\Gamma_{\varnothing}(0)\right)>\sum_{\ell(s)=J} \pi(s) v^{\prime}\left(\frac{R}{\theta^{*}(p)+\theta^{*}(s)+1}\left(y-\sum_{q \leqslant s} \Gamma_{q}(1)\right)\right) .
$$

Finally, by assumption (35) (non-increasing absolute risk aversion), there is a function $\phi: \mathfrak{R} \rightarrow \mathfrak{R}_{+}$such that $\phi^{\prime}<0, \phi^{\prime \prime}>0$, and for all $c, v^{\prime}(c)=\phi(v(c)) .{ }^{12}$ Thus (A.8) can be rewritten as

$$
\begin{aligned}
& \phi\left(v\left(\Gamma_{\varnothing}(0)\right)\right) \\
& \quad>\sum_{\ell(s)=J} \pi(s) \phi\left(v\left(\frac{R}{\theta^{*}(p)+\theta^{*}(s)+1}\left(y-\sum_{q \leqslant s} \Gamma_{q}(1)\right)\right)\right) .
\end{aligned}
$$

\footnotetext{
${ }^{12}$ In fact, function $\phi(u) \equiv v^{\prime}\left(v^{-1}(u)\right)$ will do the job.
} 
By convexity of $\phi$ and Jensen's inequality,

$$
\begin{aligned}
& \phi\left(v\left(\Gamma_{\varnothing}(0)\right)\right) \\
& \quad>\phi\left(\sum_{\ell(s)=J} \pi(s) v\left(\frac{R}{\theta^{*}(p)+\theta^{*}(s)+1}\left(y-\sum_{q \leqslant s} \Gamma_{q}(1)\right)\right)\right) .
\end{aligned}
$$

Since $\phi^{\prime}<0$, this inequality implies that

$$
v\left(\Gamma_{\varnothing}(0)\right)<\sum_{\ell(s)=J} \pi(s) v\left(\frac{R}{\theta^{*}(p)+\theta^{*}(s)+1}\left(y-\sum_{q \leqslant s} \Gamma_{q}(1)\right)\right),
$$

which is the desired conclusion.

\section{References}

[1] J. Bryant, A model of reserves, bank runs, and deposit insurance, J. Banking Finance 43 (1980) 749-761.

[2] C. Calomiris, C. Kahn, The role of demandable debt in structuring optimal banking arrangements, Amer. Econ. Review 81 (1991) 497-513.

[3] D. Diamond, Financial intermediation and delegated monitoring, Rev. Econ. Stud. 52 (1984) 393-414.

[4] D. Diamond, P. Dybvig, Bank runs, deposit insurance, and liquidity, J. Polit. Econ. 91 (1983) 401-419.

[5] E. Green, P. Lin, Diamond and Dybvig's classical theory of financial intermediation: What's missing?, Fed. Reserve Bank Minneapolis Quart. Rev. 24 (2000) 3-13.

[6] C. Jacklin, Demand deposits, trading restrictions, and risking sharing, in: E. Prescott, N. Wallace (Eds.), Contractual Arrangements for Intertemporal Trade, University of Minnesota Press, Minneapolis, 1987.

[7] S. Krasa, A. Villamil, Monitoring the monitor: an incentive structure for a financial intermediary, J. Econ. Theory 57 (1992) 197-221.

[8] P. Lin, Absence of strategic dominance under the sequential service constraint, Mimeo, Lingnan University, 2001.

[9] R. Myerson, Game Theory: Analysis of Conflict, Harvard University Press, Cambridge, MA, 1991.

[10] J. Peck, K. Shell, Equilibrium bank runs, Mimeo, The Ohio State University, Cornell University, June 2001.

[11] N. Wallace, Another attempt to explain an illiquid banking: the Diamond-Dybvig model with sequential service taken seriously, Fed. Reserve Bank Minneapolis Quart. Rev. 12 (1988) 3-16.

[12] N. Wallace, A Banking model in which partial suspension is best, Fed. Reserve Bank Minneapolis Quart. Rev. 14 (1990) 11-23. 